\title{
Symmetric Integer Matrices Having Integer Eigenvalues
}

\author{
Lei Cao' ${ }^{1}$ and Selcuk Koyuncu ${ }^{2}$ \\ ${ }^{1}$ Department of Mathematics, Georgian Court University, Lakewood, NJ 08701, USA \\ ${ }^{2}$ Department of Mathematics, University of North Georgia, Gainesville, GA 30566, USA
}

Correspondence should be addressed to Selcuk Koyuncu; skoyuncu@ung.edu

Received 13 September 2016; Revised 13 October 2016; Accepted 18 October 2016

Academic Editor: Niansheng Tang

Copyright (C) 2016 L. Cao and S. Koyuncu. This is an open access article distributed under the Creative Commons Attribution License, which permits unrestricted use, distribution, and reproduction in any medium, provided the original work is properly cited.

We provide characterization of symmetric integer matrices for rank at most 2 that have integer spectrum and give some constructions for such matrices of rank 3. We also make some connection between Hanlon's conjecture and integer eigenvalue problem.

\section{Introduction}

The study of matrices with integer entries combines linear algebra, number theory, and group theory (the study of arithmetic groups). It was shown that the eigenvalues of symmetric matrices over the integers $\mathbb{Z}$ stem from as to what algebraic integers occur as eigenvalues for the incidence matrix of a graph (see [1]). Integer eigenvalues of a nonsymmetric matrix with entries as certain simple functions are presented in [2]. A graph is Laplacian integral if the spectrum of its Laplacian matrix consists entirely of integers. A number of papers on Laplacian matrices investigate the class of Laplacian integral graphs (see [3-5]). Integer matrices that arise from Laplacians are connected to the three-dimensional Heisenberg Lie algebra and the eigenvalues and eigenvectors were explicitly given for the subclass of these matrices (see [6]). An interesting class of matrices called $B_{n}$ was introduced in [7]; the most interesting property of the $B_{n}$-class is that the spectra of the matrices consist of the consecutive integers $\{0,1, \ldots, n-1\}$; that is, the eigenvalues do not depend on the values of the elements of $B \in B_{n}$. In this paper, we characterize all symmetric integer matrices for rank at most 2 that have integer spectrum and give some constructions for such matrices of rank 3 . We also open a discussion on the fact that integer eigenvalue problem has strong connection with Hanlon's conjecture (see [6]). We provide some examples and conjectures that relate these two problems.
We start with some basic definitions from linear algebra. Let $A$ be a square matrix of size $n$ and let $\lambda$ be a scalar quantity. Then $P_{A}(\lambda)=\operatorname{det}(A-\lambda I)$ is called the characteristic polynomial of $A$. It is clear that the characteristic polynomial is an $n$th degree polynomial in $\lambda$ and $\operatorname{det}(A-\lambda I)=0$ will have $n$ (not necessarily distinct) solutions for $\lambda$. The values of $\lambda$ that satisfy $\operatorname{det}(A-\lambda I)=0$ are the characteristic roots or eigenvalues of $A$. An $n \times n$ matrix $A$ is called real symmetric if $A^{T}$, the transpose of $A$, coincide with $A$. If $A=\left[a_{i j}\right]$ is an $m \times n$ matrix and $B=\left[b_{i j}\right]$ is an $p \times q$ matrix, then the tensor product of $A$ and $B$, denoted by $A \otimes B$, is the $m p \times n q$ matrix and is defined as

$$
A \otimes B=\left(\begin{array}{ccc}
a_{11} B & \cdots & a_{1 n} B \\
\vdots & \ddots & \vdots \\
a_{m 1} B & \cdots & a_{m n} B
\end{array}\right) .
$$

If $A$ is $n \times n$ and $B$ is $m \times m$, then the Kronecker sum (or tensor sum) of $A$ and $B$, denoted by $A \oplus B$, is the $m n \times m n$ matrix of the form $\left(I_{m} \otimes A\right)+\left(B \otimes I_{n}\right)$. Let $M_{n}(\mathbb{Z})$ be the set of all $n \times n$ symmetric matrices with integer entries.

Let $A$ be an $n \times n$ matrix and $A_{[k]}$ be the sum of all $k$ th order principal minors of $A$. Then all coefficients in the characteristic polynomial of $A$ can be expressed by $A_{[k]}$ for $k=1,2, \ldots, n$. In particular, $A_{[1]}=\operatorname{trace}(A)$ and $A_{[n]}=$ $\operatorname{det}(A)$. 
Lemma 1. Let $A \in M_{n}(\mathbb{R})$ with eigenvalues $\lambda_{1}, \lambda_{2}, \ldots, \lambda_{n}$. The characteristic polynomial of $A$ is given by

$$
\begin{aligned}
p_{A}(\lambda) & =\operatorname{det}(\lambda I-A) \\
& =\lambda^{n}+c_{n-1} \lambda^{n-1}+c_{n-2} \lambda^{n-2}+\cdots+c_{1} \lambda+c_{0} .
\end{aligned}
$$

Then

$$
c_{i}=A_{[n-i]}, \quad i=0, \ldots, n-1 .
$$

Lemma 2. Let $A \in M_{n}(\mathbb{Z})$ with rank 1 . Then $A$ has integer eigenvalues.

We now present the characterization of all symmetric integer matrices for rank at most 2 that have integer spectrum.

\section{The Rank 2 Case}

Theorem 3. Let $A \in M_{n}(\mathbb{Z})$ with rank 2. Then $A$ has integer eigenvalues if and only if there exist two integers $m$ and $n$ such that $\operatorname{trace}(A)=m+n$ and $A_{[2]}=m n$ where $A_{[2]}$ is the sum of determinants of all 2 nd order principal minors of $A$.

Proof. Since $A$ has rank 2, the characteristic polynomial of $A$ has the form

$$
\lambda^{n}-\operatorname{trace}(A) \lambda^{n-1}+A_{[2]} \lambda^{n-2}
$$

It is clear that the two nonzero eigenvalues of $A$ are

$$
\begin{aligned}
& \lambda_{1}=\frac{\operatorname{trace}(A)+\sqrt{\operatorname{trace}(A)^{2}-4 A_{[2]}}}{2}, \\
& \lambda_{2}=\frac{\operatorname{trace}(A)-\sqrt{\operatorname{trace}(A)^{2}-4 A_{[2]}}}{2} .
\end{aligned}
$$

" $\Leftarrow$ " Suppose there exist integers $m$ and $n$ such that $\operatorname{trace}(A)=m+n$ and $A_{[2]}=m n$. Then it follows that

$$
\begin{aligned}
& \lambda_{1}=m, \\
& \lambda_{2}=n .
\end{aligned}
$$

“ $\Rightarrow$ ” If all eigenvalues of $A$ are integers, then there exists an integer $k$ such that

$$
\operatorname{trace}(A)^{2}-4 A_{[2]}=k^{2} .
$$

Letting

$$
\begin{aligned}
& m=\frac{\operatorname{trace}(A)+k}{2}, \\
& m=\frac{\operatorname{trace}(A)-k}{2}
\end{aligned}
$$

and using (7), the difference of $\operatorname{trace}(A)^{2}$ and $k^{2}$ is $4 A_{[2]}$ which is even, so either both $\operatorname{trace}(A)$ and $k$ are even or both $\operatorname{trace}(A)$ and $k$ are odd, and hence both $m$ and $n$ are integers.
Lemma 4. Let $A=\left(\begin{array}{cc}B & C \\ D & E\end{array}\right)$. If $E$ is invertible and $D E=E D$, then $\operatorname{det}(A)=\operatorname{det}(B E-C D)$.

Theorem 5. Let $A=\left(\begin{array}{ll}B & C \\ C\end{array}\right)$. Suppose both $B$ and $C$ are integer matrices of order $n$ with integer eigenvalues. If $B$ and $C$ commute, then $A$ has integer eigenvalues.

Proof. According to Lemma 4,

$$
\begin{aligned}
\operatorname{det}\left(\lambda I_{2 n}-A\right) & =\operatorname{det}\left(\left(\lambda I_{2 n}-B\right)^{2}-C^{2}\right) \\
& =\operatorname{det}\left(\left(\lambda I_{2 n}-B-C\right)\left(\lambda I_{2 n}-B+C\right)\right) .
\end{aligned}
$$

Since $B$ and $C$ commute, they can be diagonalized simultaneously and hence all eigenvalues of both $B+C$ and $B-C$ are integers.

Lemma 6. Let

$$
A=\left(\begin{array}{ccccc}
B & I & O & \cdots & O \\
O & B & I & O & \vdots \\
\vdots & \ddots & \ddots & \ddots & O \\
O & \cdots & O & B & I \\
O & \cdots & \cdots & O & B
\end{array}\right) \in M_{2 n}(\mathbb{Z}) .
$$

If $B=\left(\begin{array}{ll}a & b \\ b & a\end{array}\right) \in M_{2}(\mathbb{Z})$, then $A$ has integer eigenvalues.

Proof. Define

$$
J=\left(\begin{array}{ccccc}
0 & 1 & 0 & \cdots & 0 \\
0 & 0 & 1 & 0 & \vdots \\
\vdots & \ddots & \ddots & \ddots & 0 \\
0 & \cdots & 0 & 0 & 1 \\
0 & \cdots & \cdots & 0 & 0
\end{array}\right) \in M_{n}(\mathbb{Z})
$$

Then we can write $A$ as

$$
A=\left(I_{n} \otimes B\right)+\left(J \otimes I_{2}\right)=B \oplus J .
$$

Since $B$ and $J$ have integer eigenvalues, then $A=B \oplus J$ has also integer eigenvalues.

We now give some constructions for all symmetric matrices of rank 3 that has integer spectrum.

\section{The Rank 3 Case}

Theorem 7. Let $A \in M_{n}(\mathbb{Z})$ be symmetric integer matrix with rank 3. If one of the following cases holds, then $A$ has integer eigenvalues.

(i) One of the eigenvalues of $A$ is 1 or -1 and there exists a positive integer $k$ such that

$$
\left[A_{[3]}-A_{[2]}\right]^{2}+4 A_{[3]}=k^{2} \text {. }
$$


(ii) All nonzero eigenvalues of $A$ are the same and

$$
\begin{aligned}
& A_{[2]}=\frac{(\operatorname{trace}(A))^{2}}{3}, \\
& A_{[3]}=\frac{(\operatorname{trace}(A))^{3}}{27} .
\end{aligned}
$$

(iii) One of the nonzero eigenvalues of $A$ has multiplicity two and there exists a positive integer such that

$$
(\operatorname{trace}(A))^{2}-3 A_{[2]}=k^{2} \text {. }
$$

(iv) The trace of $A$ is equal to zero and there exists a positive integer $k$ and integers $m, n$ such that

$$
\begin{aligned}
k & =\sqrt{\frac{\left(A_{[3]}\right)^{2}}{4}+\frac{\left(A_{[2]}\right)^{3}}{27},} \\
m^{3} & =\frac{A_{[3]}}{2}+k, \\
n^{3} & =\frac{A_{[3]}}{2}-k .
\end{aligned}
$$

In fact, one of eigenvalues is $m+n$.

Proof. Since the rank of $A$ is 3, the characteristics polynomial of $A$ can be written as

$$
\begin{aligned}
P_{A}(\lambda) & =\lambda^{n}-\operatorname{trace}(A) \lambda^{n-1}+A_{[2]} \lambda^{n-2}-A_{[3]} \lambda^{n-3} \\
& =\lambda^{n-3}\left(\lambda^{3}-\operatorname{trace}(A) \lambda^{2}+A_{[2]} \lambda-A_{[3]}\right) .
\end{aligned}
$$

(i) Suppose that one of the eigenvalues of $A$ is $\lambda=1$. By substituting this eigenvalue in (17), one obtains

$$
A_{[3]}-A_{[2]}=1-\operatorname{trace}(A) .
$$

In addition, (17) can be factored as

$$
P_{A}(\lambda)=\lambda^{n-3}(\lambda-1)\left[\lambda^{2}+(1-\operatorname{trace}(A)) \lambda+A_{[3]}\right] .
$$

By Theorem 3, the quadratic factor has integer roots if and only if there exists a positive integer $k$, such that

$$
(1-\operatorname{trace}(A))^{2}+4 A_{[3]}=k^{2} .
$$

Now combining (18) and (20) yields

$$
\left(A_{[3]}-A_{[2]}\right)^{2}+4 A_{[3]}=k^{2} .
$$

And in fact, the other eigenvalues are

$$
\begin{aligned}
& \lambda_{2}=\frac{1-\operatorname{trace}(A)+k}{2}, \\
& \lambda_{3}=\frac{-1+\operatorname{trace}(A)-k}{2}
\end{aligned}
$$

which are integers because either both $1-\operatorname{trace} A$ and $k$ are even or both of them are odd by (20).

(ii) Let $\tilde{\lambda}$ be the only nonzero eigenvalue of $A$. Then

$$
\begin{aligned}
P_{A}(\lambda) & =\lambda^{n-3}(\lambda-\tilde{\lambda})^{3} \\
(\lambda-\tilde{\lambda})^{3} & =\lambda^{3}-\operatorname{trace}(A) \lambda^{2}+A_{[2]} \lambda-A_{[3]} .
\end{aligned}
$$

By comparing the coefficients on both sides of (24), one obtains

$$
\begin{aligned}
\operatorname{trace}(A) & =3 \tilde{\lambda}, \\
A_{[2]} & =3 \tilde{\lambda}^{2}, \\
A_{[3]} & =\tilde{\lambda}^{3} .
\end{aligned}
$$

Thus

$$
\begin{aligned}
& A_{[2]}=\frac{(\operatorname{trace}(A))^{2}}{3}, \\
& A_{[3]}=\frac{(\operatorname{trace}(A))^{3}}{27} .
\end{aligned}
$$

(iii) Suppose that $A$ has two nonzero eigenvalues $\lambda_{1}$ and $\lambda_{2}$ with multiplicity one and two, respectively. Then the characteristic polynomial of $A$ can be written as

$$
P_{A}(\lambda)=\lambda^{n-3}\left(\lambda-\lambda_{1}\right)^{2}\left(\lambda-\lambda_{2}\right),
$$

and hence

$$
\begin{aligned}
\left(\lambda-\lambda_{1}\right)^{2}\left(\lambda-\lambda_{2}\right)= & \lambda^{3}-\operatorname{trace}(A) \lambda^{2}+A_{[2]} \lambda \\
& -A_{[3]} .
\end{aligned}
$$

By comparing the coefficients on both sides of (28), one obtains

$$
\begin{aligned}
\operatorname{trace}(A) & =2 \lambda_{1}+\lambda_{2}, \\
A_{[2]} & =\lambda_{1}^{2}+2 \lambda_{1} \lambda_{2}, \\
A_{[3]} & =\lambda_{1}^{2} \lambda_{2} .
\end{aligned}
$$

In addition, since $\lambda_{1}$ has multiplicity two, both $P_{A}\left(\lambda_{1}\right)$ and its derivative $P_{A}^{\prime}\left(\lambda_{1}\right)$ are equal to zero and hence

$$
3 \lambda_{1}^{2}-2 \operatorname{trace}(A) \lambda_{1}+A_{[2]}=0 \text {. }
$$

Now taking the derivative of both sides of (28), we get

$$
\begin{aligned}
3 \lambda^{2} & -2 \operatorname{trace}(A) \lambda+A_{[2]} \\
& =\left(\lambda-\lambda_{1}\right)^{2}+2\left(\lambda-\lambda_{1}\right)\left(\lambda-\lambda_{2}\right) .
\end{aligned}
$$


Since (31) is quadratic and has one integer root $\lambda_{1}$, then the other root must be rational. Thus there exists a positive integer $k$ such that

$$
4(\operatorname{trace}(A))^{2}-12 A_{[2]}=(2 k)^{2}
$$

which yields that

$$
(\operatorname{trace}(A))^{2}-3 A_{[2]}=k^{2}
$$

(iv) Denote the nonzero eigenvalues of $A$ by $\lambda_{1}, \lambda_{2}$, and $\lambda_{3}$. We have $\lambda_{3}=-\left(\lambda_{1}+\lambda_{2}\right)$ due to zero trace. Also, we have the following equations

$$
\begin{aligned}
\lambda_{1}^{2}+\lambda_{1} \lambda_{2}+\lambda_{2}^{2} & =-A_{[2]}, \\
\lambda_{1}^{2} \lambda_{2}+\lambda_{1} \lambda_{2}^{2} & =-A_{[3]} .
\end{aligned}
$$

Multiplying (34) by $\lambda_{1}$ yields that

$$
\lambda_{1}^{3}+\lambda_{1}^{2} \lambda_{2}+\lambda_{1} \lambda_{2}^{2}=-A_{[2]} \lambda_{1}
$$

Subtracting (35) from (36), one obtains

$$
\lambda_{1}^{3}+A_{[2]} \lambda_{1}-A_{[3]}=0 .
$$

Note that the following

$$
\begin{aligned}
& \sqrt[3]{\frac{A_{[3]}}{2}+\sqrt{\frac{\left(A_{[3]}\right)^{2}}{4}+\frac{\left(A_{[3]}\right)^{3}}{27}}} \\
& +\sqrt[3]{\frac{A_{[3]}}{2}-\sqrt{\frac{\left(A_{[3]}\right)^{2}}{4}+\frac{\left(A_{[2]}\right)^{3}}{27}}}=\sqrt[3]{\frac{A_{[3]}}{2}+k} \\
& +\sqrt[3]{\frac{A_{[3]}}{2}-k}=m+n
\end{aligned}
$$

is always a solution of (36). To see this, first note that

$$
\begin{aligned}
m \cdot n= & \sqrt[3]{\frac{A_{[3]}}{2}+k} \cdot \sqrt[3]{\frac{A_{[3]}}{2}-k} \\
= & \sqrt[3]{\frac{\left(A_{[3]}\right)^{2}}{4}-\frac{\left(A_{[3]}\right)^{2}}{4}-\frac{\left(A_{[2]}\right)^{3}}{27}}=-\frac{A_{[3]}}{3}, \\
(m+n)^{3}= & m^{3}+3 m^{2} n+3 m n^{2}+n^{3} \\
= & \left(\frac{A_{[3]}}{2}+k\right)+3 m n(m+n)+\left(\frac{A_{[3]}}{2}-k\right) \\
= & \left(\frac{A_{[3]}}{2}+k\right)-\frac{A_{[2]}}{3} \cdot(m+n) \\
& +\left(\frac{A_{[3]}}{2}-k\right)=-\frac{A_{[2]}}{3} \cdot(m+n)+A_{[3]} .
\end{aligned}
$$

Lemma 8. Let

$$
F=\left(\begin{array}{cccc}
1 & 1 & \cdots & 1 \\
1 & 1 & \cdots & 1 \\
\vdots & \vdots & \ddots & \vdots \\
1 & 1 & \cdots & 1
\end{array}\right) \in M_{m}
$$

and let $A=(F \otimes B)+\left(C \otimes I_{m}\right)$. Then $A$ has integer eigenvalues if $B C=C B$ and both $B$ and $C$ have integer eigenvalues.

Proof. Since $B$ and $C$ commute, they can be diagonalized simultaneously. Without loss of generality, suppose

$$
\begin{aligned}
& B=\left(\begin{array}{llll}
b_{1} & & & \\
& b_{2} & & \\
& & \ddots & \\
& & & b_{n}
\end{array}\right), \\
& C=\left(\begin{array}{llll}
c_{1} & & \\
& c_{2} & \\
& & \ddots & \\
& & & c_{n}
\end{array}\right) .
\end{aligned}
$$

Note that $A$ has eigenvalues $b_{i}+(n-1) c_{i}$ with multiplicity 1 for $i=1,2, \ldots, n$ and $A$ has eigenvalue $b_{i}-c_{i}$ with multiplicities $m-1$ for $i=1,2, \ldots, n$ since $\left(b_{i}-c_{i}\right) I-A$ has rank $(n-1) m$.

Lemma 9. Let $A \in M_{n}(\mathbb{Z})$. If $A=A_{1} \otimes A_{2} \otimes \cdots \otimes A_{k}$ where each $A_{i}$ has integer eigenvalues and $A_{i} \in M_{i}(\mathbb{Z}), i=1, \ldots, k$, then $A$ has integer eigenvalues.

Proposition 10. Let $A^{T}=A \in M_{3}(\mathbb{Z})$ and $B=\left(\begin{array}{ll}a & b \\ b & a\end{array}\right) \in$ $M_{2}(\mathbb{Z})$. Suppose both $A$ and $B$ have integer eigenvalues. Then $A \oplus B$ has integer eigenvalues.

In this section, we open a discussion on possible connection between integer eigenvalue problem and Hanlon's conjecture. We support our approach with some examples and adopt the notation used in [6].

\section{Connection to Hanlon's Conjecture}

Definition 11. Let $a, b$, and $k$ be nonnegative integers with $a \leq$ $k+1$ and $b \leq k+1$. Let $\Omega_{k}(a, b)$ be the set of pairs $(U, V)$ such that $U$ is an $A$-subset of $k_{0}$ and $V$ is a $B$-subset of $k_{0}$, where $k_{0}=\{0,1, \ldots, k\}$ and $k$ is a nonnegative integer. Define the weight of a pair $(U, V)$ to be

$$
\mathbb{W}(U, V)=\sum_{u \in U} u+\sum_{v \in V} v
$$

Let $\Omega_{k}(a, b, w)$ be the set of pairs $(U, V)$ such that $\mathbb{W}(U, V)=$ $w$.

Example 12. Let $a=2, b=1$, and $w=4$. If $k=$ 2 , then the ordered basis is $\Omega_{2}(2,1,4)=\{(12,1),(02,2)\}$. 
If $k=3$, the ordered basis is $\Omega_{2}(2,1,4)=\{(13,0),(03,1)$, $(12,1),(02,2),(01,3)\}$. In this case, if $k=0$ then $\Omega_{2}(2,1,4)=$ $\emptyset$.

We define a matrix $T_{k}(a, b, w)$ with respect to corresponding basis $\Omega_{k}(a, b, w)$. We need the following definition in order to define the matrix $T_{k}(a, b, w)$.

Definition 13. Let $(U, V)$ and $(X, Y)$ be elements of $\Omega_{k}(a, b, w)$, and let $(u, v, z)$ be a triple with $u \in U$, $v \in V$ and $z$ is any integer. we say that $(U, V)$ and $(X, Y)$ are $(u, v, z)$-neighbors if

(1) $X=(U \backslash\{u\}) \cup\{u+z\}$,

(2) $Y=(Y \backslash\{v\}) \cup\{v-z\}$,

(3) $u+v \leq k$.

In [6] (Conjecture 1.12), it was conjectured that the eigenvalues $T_{k}(a, b, w)$ are nonnegative integers. Let us present some examples.

Example 14. $T_{2}(2,1,4)$ with respect to the ordered basis $\{(12,1),(02,2)\}$ is

$$
\left(\begin{array}{ll}
2 & 1 \\
1 & 2
\end{array}\right) .
$$

Theorem 3 guarantees that both eigenvalues of $T_{2}(2,1,4)$ are integers.

Example 15. $T_{3}(2,1,4)$ with respect to the ordered basis

$$
\{(13,0),(03,1),(12,1),(02,2),(01,3)\}
$$

is

$$
\left(\begin{array}{ccccc}
2 & 1 & 1 & 0 & -1 \\
1 & 2 & 0 & 1 & 1 \\
1 & 0 & 2 & 1 & -1 \\
0 & 1 & 1 & 2 & 1 \\
-1 & 1 & -1 & 1 & 2
\end{array}\right)
$$

If the last row and column are deleted, the resulting matrix is in the form of block matrix given in Theorem 5. The eigenvalues of $(1,1)$-block are 1,3 and the eigenvalues of $(2,2)$-block are both 1 with multiplicity 2 . The eigenvalues of $T_{2}(2,1,4)$ are $0,0,2,4,4$. Notice that these eigenvalues can also be obtained from the sum and difference of $(1,1)$ and $(1,2)$ blocks with given multiplicities.

Example 16. $T_{4}(2,1,4)$ with respect to the ordered basis

$$
\{(04,0),(13,0),(03,1),(12,1),(02,2),(01,3)\}
$$

is

$$
\left(\begin{array}{cccccc}
2 & 0 & 1 & 0 & 1 & 1 \\
0 & 2 & 1 & 1 & 0 & -1 \\
1 & 1 & 2 & 0 & 1 & 1 \\
0 & 1 & 0 & 2 & 1 & -1 \\
1 & 0 & 1 & 1 & 2 & 1 \\
1 & -1 & 1 & -1 & 1 & 2
\end{array}\right)
$$

The eigenvalues of $T_{4}(2,1,4)$ are $0,0,1,2,4$, and 5 . We do not observe an obvious connection in this example.

Example 17. $T_{3}(3,2,5)$ with respect to the ordered basis

$$
\{(012,02)\}
$$

is

$$
\text { (4). }
$$

Example 18. $T_{3}(2,2,4)$ with respect to the ordered basis

$$
\{(13,01),(12,02),(02,12),(01,13)\}
$$

is

$$
\left(\begin{array}{llll}
3 & 1 & 0 & 1 \\
1 & 3 & 1 & 0 \\
0 & 1 & 3 & 1 \\
1 & 0 & 1 & 3
\end{array}\right)
$$

Notice that the matrix $T_{3}(2,2,4)$ is in block form given in Theorem 5 and its eigenvalues are the eigenvalues of the sum and difference of $(1,1)$ and $(2,2)$-blocks with given multiplicities. In this case the eigenvalues are 1, 3, 3, and 5.

Example 19. $T_{3}(2,2,6)$ with respect to the ordered basis

$$
\begin{aligned}
& \{(23,01),(13,02),(03,12),(03,03),(12,12),(12,03), \\
& \quad(02,13),(01,23)\}
\end{aligned}
$$

is

$$
\left(\begin{array}{cccccccc}
3 & 1 & -1 & 1 & 1 & -1 & 1 & 0 \\
1 & 3 & 1 & 1 & 1 & 1 & 0 & -1 \\
-1 & 1 & 2 & 0 & 0 & 0 & 1 & -1 \\
1 & 1 & 0 & 3 & 0 & 0 & 1 & 1 \\
1 & 1 & 0 & 0 & 3 & 0 & 1 & 1 \\
-1 & 1 & 0 & 0 & 0 & 2 & 1 & -1 \\
1 & 0 & 1 & 1 & 1 & 1 & 3 & 1 \\
0 & 1 & -1 & 1 & 1 & -1 & 1 & 3
\end{array}\right) .
$$

Note that some examples given above follow directly from the theory we have provided and some do not. We aim to search more general connections in a followup paper. In [6], 
the author search for algebraic expression for the eigenvalues. We use the same notation and state the conjecture. For each $a, b, w, k$, and each nonnegative integer $r$, let $\mu_{k}(a, b, w ; r)$ denote the multiplicity of $r$ as an eigenvalue of $T_{k}(a, b, w)$. Let

$$
\mu_{k}(a, b ; r)=\sum_{w} \mu_{k}(a, b, w ; r) .
$$

Let $M_{k}(x, y, \lambda)$ be the following generating function for the numbers $\mu_{k}(a, b ; r)$ :

$$
M_{k}(x, y, \lambda)=\sum_{a, b, r} \mu_{k}(a, b ; r) x^{a} y^{b} \lambda^{r}
$$

It was conjectured in [6] (Conjecture 1.14) that $M_{k}(x$, $y, \lambda)=\prod_{i=0}^{k}\left(1+x+y+\lambda^{i+1} x y\right)$. This conjecture is still open in general. We hope to find more obvious connections and use them to solve given conjectures in this paper.

\section{Competing Interests}

Lei Cao and Selcuk Koyuncu declare that there is no conflict of interests regarding the publication of this paper.

\section{References}

[1] D. R. Estes, "Eigenvalues of symmetric integer matrices," Journal of Number Theory, vol. 42, no. 3, pp. 292-296, 1992.

[2] L. Bondesson and I. Traat, "A nonsymmetric matrix with integer eigenvalues," Linear and Multilinear Algebra, vol. 55, no. 3, pp. 239-247, 2007.

[3] F. Harary and A. J. Schwenk, "Which graphs have integral spectra?" in Graphs and Combinatorics: Proceedings of the Capital Conference on Graph Theory and Combinatorics at the George Washington University June 18-22, 1973, R. A. Bari and F. Harary, Eds., vol. 406 of Lecture Notes in Mathematics, pp. 45-51, Springer, Berlin, Germany, 1974.

[4] S. Kirkland, "Constructably Laplacian integral graphs," Linear Algebra and Its Applications, vol. 423, no. 1, pp. 3-21, 2007.

[5] W. So, "Rank one perturbation and its application to the Laplacian spectrum of a graph," Linear and Multilinear Algebra, vol. 46, no. 3, pp. 193-198, 1999.

[6] P. Hanlon, "Some remarkable combinatorial matrices," Journal of Combinatorial Theory, Series A, vol. 59, no. 2, pp. 218-239, 1992.

[7] T. von Rosen and D. von Rosen, "On a class of singular nonsymmetric matrices with nonnegative integer spectra," in Algebraic Methods in Statistics and Probability II, M. A. G. Viana and H. P. Wynn, Eds., vol. 516, pp. 319-325, American Mathematical Society, Providence, RI, USA, 2010. 


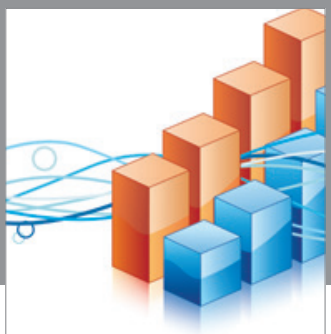

Advances in

Operations Research

vatem alat4

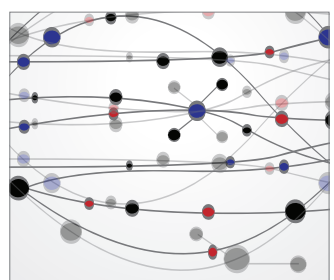

\section{The Scientific} World Journal
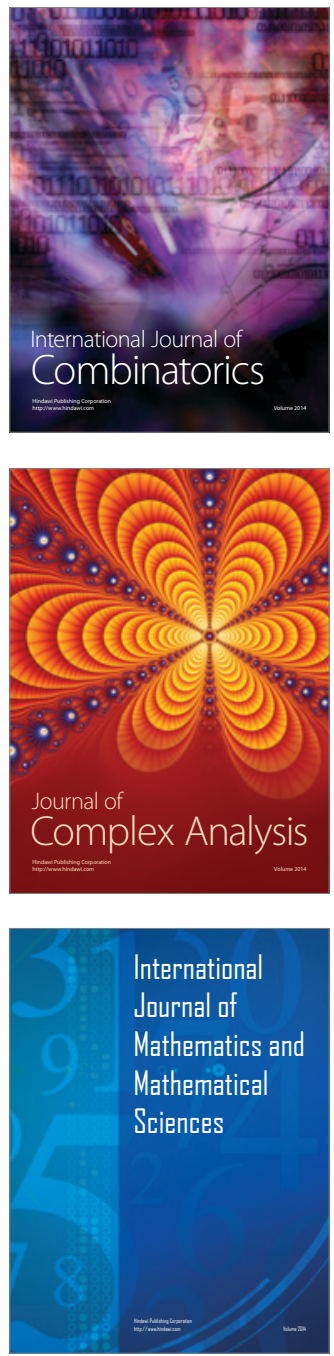
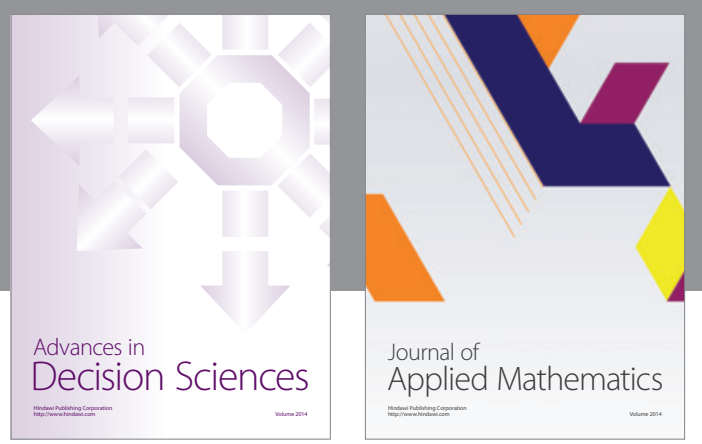

Algebra

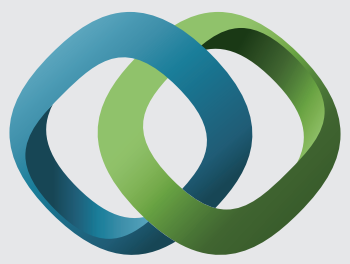

\section{Hindawi}

Submit your manuscripts at

http://www.hindawi.com
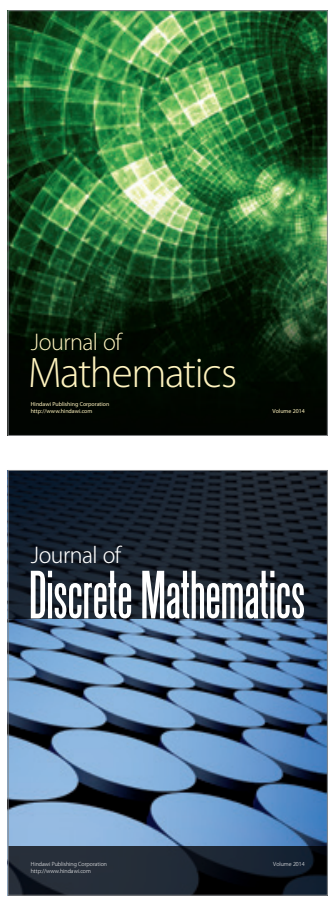

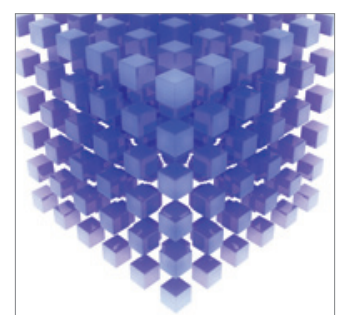

Mathematical Problems in Engineering
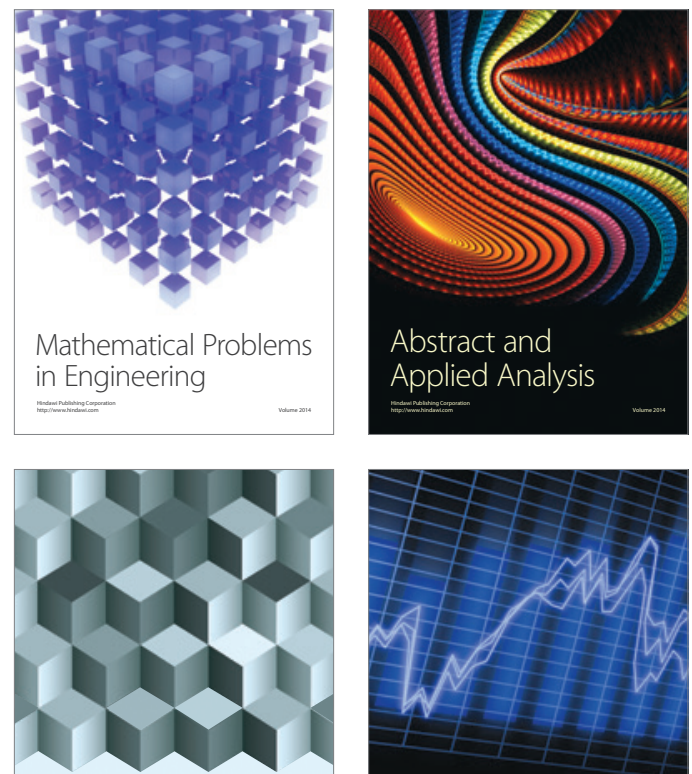

Journal of

Function Spaces

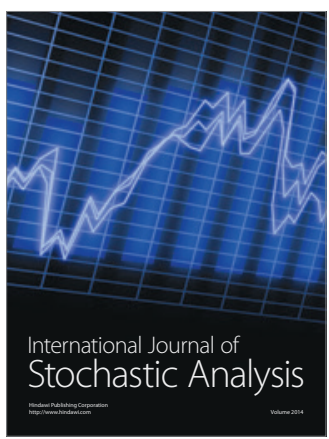

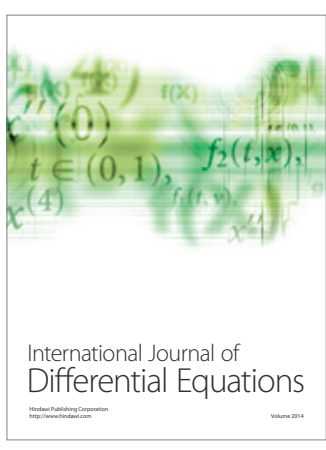
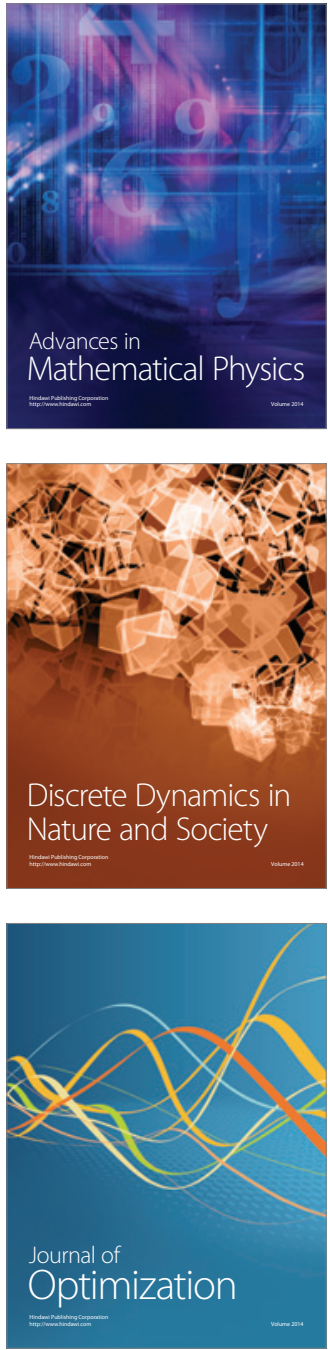